\title{
Autoantibodies to IA-2 $\beta$ improve diabetes risk assessment in high-risk relatives
}

\author{
P. Achenbach • E. Bonifacio • A. J. K. Williams • \\ A. G. Ziegler • E. A. M. Gale • P. J. Bingley • \\ and the ENDIT Group
}

Received: 17 August 2007 / Accepted: 10 December 2007 / Published online: 9 January 2008

(C) Springer-Verlag 2007

\begin{abstract}
Aims/hypothesis The aim of this study was to evaluate the prognostic significance of autoantibodies to IA-2 $\beta$ (IA2 $\beta A$ ) in a large, well-characterised population of islet cell antibody (ICA)-positive relatives followed for 5 years in the European Nicotinamide Diabetes Intervention Trial.

Methods Autoantibodies to insulin (IAA), glutamate decarboxylase (GADA) and IA-2 (IA2A) were measured in 549 participants at study entry, and IA2A-positive samples tested for IA2 $\beta$ A. First-phase insulin response (FPIR) and oral glucose tolerance were determined at baseline.

Results Of 212 ICA/IA2A-positive participants (median age 12.1 years; $57 \%$ male), 113 developed diabetes (5 year cumulative risk $56 \%$ ), and 148 were also GADA-positive and IAA-positive (4Ab-positive). IA2 $\beta$ A were detected in 137 (65\%) ICA/IA2A-positive participants and were associated with an increased 5 year diabetes risk (IA2 $\beta A$ positive 65 vs $39 \%$ in IA $2 \beta A$-negative, $p=0.0002$ ). The
\end{abstract}

Electronic supplementary material The online version of this article (doi:10.1007/s00125-007-0912-9) contains a list of members of the ENDIT Group, which is available to authorised users.

P. Achenbach · E. Bonifacio • A. G. Ziegler

Diabetes Research Institute,

Munich, Germany

A. J. K. Williams $\cdot$ E. A. M. Gale $\cdot$ P. J. Bingley $(\bowtie)$

Diabetes and Metabolism,

Department of Clinical Science at North Bristol,

University of Bristol, Southmead Hospital,

Bristol BS10 5NB, UK

e-mail: polly.bingley@bristol.ac.uk effect was most marked in $4 \mathrm{Ab}$-positive relatives $(72 \%$ vs $52 \%, p=0.003)$. Metabolic testing further refined risk assessment. Among $1014 \mathrm{Ab}$-positive relatives with IA2 $\beta \mathrm{A}$, the 5 year risk was $94 \%$ in those with a low FPIR (vs $50 \%$ in those with a normal FPIR, $p<0.0001$ ), and $95 \%$ in those with impaired glucose tolerance (IGT) (vs 66\% in those with normal glucose tolerance, $p<0.0001)$. The median time to diagnosis of 4Ab/IA2 $\beta A$-positive participants with a low FPIR was 1.5 years. Multivariate analysis confirmed IA2 $\beta$ A status, antibody number, young age, FPIR and IGT as independent determinants of risk.

Conclusions/interpretation IA2 $\beta \mathrm{A}$ are associated with a very high risk of diabetes in ICA/IA2A-positive relatives. Testing for IA2A/IA2 $\beta$ A compares favourably with the IVGTT in identifying a subgroup of autoantibody-positive relatives at increased risk. IA $2 \beta$ A determination should be added to screening protocols of future intervention trials.

Keywords First-phase insulin response - IA- $2 \beta$. Islet autoantibodies $\cdot$ Prediction $\cdot$ Type 1 diabetes

$\begin{array}{ll}\text { Abbreviations } \\ \text { Ab } & \text { antibody } \\ \text { ENDIT } & \begin{array}{l}\text { European Nicotinamide Diabetes Intervention } \\ \text { Trial }\end{array} \\ \text { FPIR } & \begin{array}{l}\text { first-phase insulin response } \\ \text { GADA }\end{array} \\ \text { autoantibodies to glutamate decarboxylase } \\ \text { HR } & \text { hazard ratio } \\ \text { IAA } & \text { autoantibodies to insulin } \\ \text { IA2A } & \text { autoantibodies to IA-2 } \\ \text { IA2 } \beta A & \text { autoantibodies to IA-2 } \beta \\ \text { ICA } & \text { islet cell antibodies } \\ \text { IGT } & \text { impaired glucose tolerance } \\ \text { IQR } & \text { interquartile range }\end{array}$




\section{Introduction}

Type 1 diabetes is preceded by the appearance of circulating islet autoantibodies that can be used for assessing diabetes risk in non-diabetic individuals [1]. The best established markers of progression are islet cell antibodies (ICA) and autoantibodies to insulin (IAA), the $65 \mathrm{kDa}$ isoform of glutamate decarboxylase (GADA) and the protein tyrosine phosphatase-like antigen IA-2 (IA2A), and diabetes risk is related to the magnitude and maturity of these autoantibody responses [1-6]. IA2A have been associated with a high risk of progression [3], and smaller studies suggest that spreading of the IA-2 antibody response to include the homologue protein IA-2 $\beta$ identifies a subgroup of relatives at particularly high risk of rapid development of diabetes [1].

We therefore investigated the role of autoantibodies to IA-2 $\beta$ (IA2 $\beta A$ ) in determining risk of progression to diabetes over a 5 year period in the large, well-characterised cohort of ICA-positive first-degree relatives of patients with type 1 diabetes recruited to the European Nicotinamide Diabetes Intervention Trial (ENDIT).

\section{Methods}

The protocol and outcome of ENDIT, and the role of additional immune, genetic and metabolic markers in assessing risk have previously been published [7, 8]. Inclusion criteria were ICA $\geq 20$ Juvenile Diabetes Foundation (JDF) units in at least one sample measured in the central laboratory, with ICA $\geq 5$ JDF units in a second sample and a nondiabetic OGTT at baseline. The protocol was approved by the research ethics committee or equivalent in each participating centre. Participants were randomised to receive either modified-release nicotinamide or placebo for 5 years, and were reviewed at baseline, 1 and 6 months after study entry and every 6 months thereafter, with an OGTT performed every 12 months. IVGTTs and OGTTs were carried out as previously described [8]. Diabetes and impaired glucose tolerance (IGT) were defined using WHO criteria.

Islet autoantibodies GADA, IA2A and IAA were measured by radiobinding assay in the baseline samples of all 549 ENDIT participants, and considered positive if $\geq 97.5$ th percentile of 2,860 schoolchildren [2]. IA2 $\beta A$ were measured in IA2A-positive samples by radiobinding assay using $\left[{ }^{35} \mathrm{~S}\right]$ methionine-labelled in vitro-translated recombinant human intracellular portion of IA-2 $\beta$ (amino acids 662-1,033 [9]).

Statistical analysis We compared continuous variables by the Mann-Whitney $U$ test and frequencies between groups by Fisher's exact test, and used Spearman's test to determine correlations. The Kaplan-Meier method was used for time-to-event survival analysis, comparing survival between groups by log-rank test. The follow-up period for each individual was calculated from the date of the baseline visit to the date of last contact or date of diagnosis of diabetes. We used Cox proportional hazards models to analyse the time-to-event outcome for individual and combined predictive markers, controlling for treatment group. The regression model included age at study entry, IA2A titre and number of islet autoantibodies as continuous variables, and presence or absence of IA2 $\beta$ A, IGT and first-phase insulin response (FPIR) above or below age-adjusted 10th percentile as categorical variables. Variables found to be significant at the $5 \%$ level in univariate analysis were included in a stepwise backward multivariate regression analysis. For all analyses, a two-tailed $p$ value of 0.05 was considered significant. All statistical analyses were performed using the Statistical Package for Social Science (SPSS 14.0, Chicago, IL, USA).

\section{Results}

Of 549 ICA-positive family members randomised in ENDIT, 229 had IA2A $\geq 97.5$ th percentile. Baseline samples for further testing were available from 212 ICA/ IA2A-positive relatives (median age 12.1 years, interquartile range [IQR] 8.4-18.1; 57\% male). Of these, 113 (53\%) progressed to diabetes over a median follow-up time of 2.1 years (IQR 1.0-3.3). The 5 year cumulative risk of diabetes in the IA2A-positive relatives was $56 \%(95 \% \mathrm{CI}$ 49-63) (Fig. 1a).

Of the 212 IA2A-positive relatives, 137 (65\%) were also IA $2 \beta A$-positive. IA2 $\beta$ A were found in 136 of $203(67 \%)$ relatives who had IAA and/or GADA in addition to ICA and IA2A, and one of nine (11\%) relatives who were positive for ICA and IA2A only $(p=0.001)$. The median age of IA2 $\beta A$-positive relatives was 11.6 years (IQR 8.0 16.3), compared with 13.2 years $(10.0-23.7)$ in IA2 $\beta A$ negative relatives $(p=0.01)$. IA $2 \beta A$ titres were positively correlated with IA2A titre $(r=0.358, p<0.0001)$, and inversely correlated with age $(r=-0.223, p=0.009)$. Among IA2A-positive relatives, IA $2 \beta A$ were strongly associated with HLA-DQ8 $(p=0.001)$ (data not shown).

The 5 year cumulative risk of diabetes in IA $2 \beta A$ positive relatives was $65 \%$ (95\% CI 57-73), compared with $39 \%(95 \%$ CI $28-50)$ in IA2 $\beta A$-negative relatives $(p=$ 0.0002 ; Fig. 1b). Risk in IA2 $\beta$ A-positive relatives could be refined by additional metabolic testing (Fig. 1c and $1 \mathrm{~d}$; Table 1). The impact of IA2 $\beta A$ on risk was most marked in relatives positive for all of the four other antibodies tested. Among 101 relatives with IA2 $\beta A$ in addition to ICA, GADA, IA2A and IAA, the overall risk of diabetes within 
a

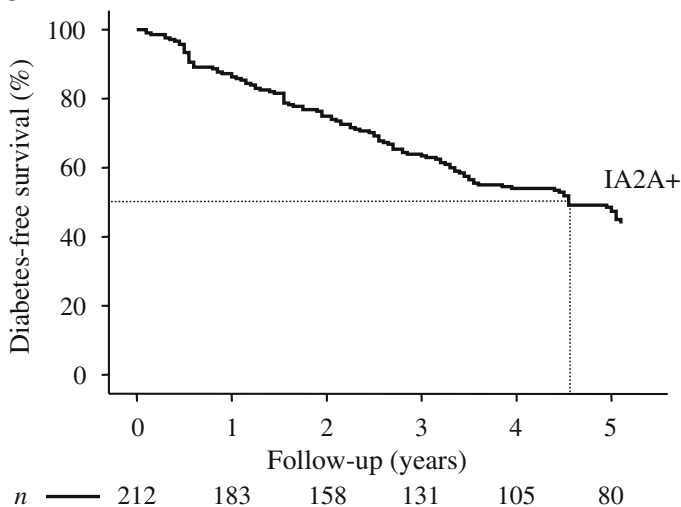

C

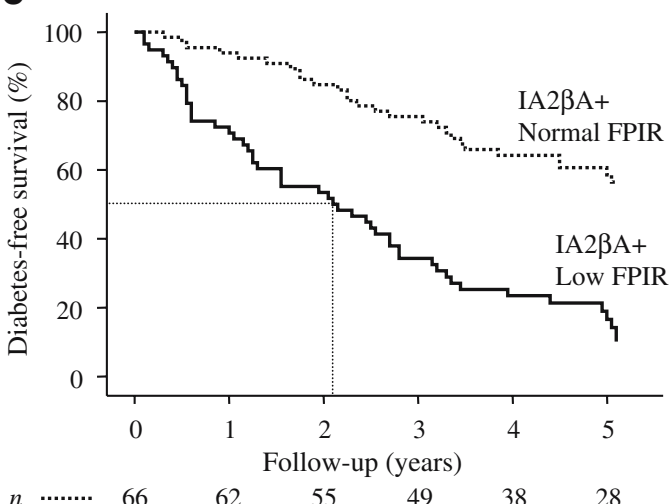

$\begin{array}{ccccccc}n-\cdots . . . . & 66 & 62 & 55 & 49 & 38 & 28 \\ - & 58 & 41 & 31 & 19 & 13 & 7\end{array}$

Fig. 1 The cumulative risk of diabetes in IA2A-positive relatives (a) in relation to presence or absence of IA2 $\beta \mathrm{A}(p=0.0002 ; \mathbf{b})$, and in IA $2 \beta A$-positive relatives in relation to presence or absence of low

5 years was $72 \%(95 \%$ CI $63-81)$, and this increased to $94 \%$ (95\% CI $86-100)$ and $95 \%$ (95\% CI 86-100) in those with low FPIR and IGT, respectively (Table 1).

Univariate analysis showed that risk varied with IA2 $\beta$ A status, number of additional islet autoantibodies, age, IA2A titre, 120 min glucose in the OGTT, and FPIR. Independent determinants of risk were loss of FPIR (hazard ratio [HR] $3.1,95 \%$ CI 2.0-4.7, $p<0.0001$ ), presence of IGT (HR 2.8, $95 \%$ CI 1.7-4.6, $p=0.0001$ ), IA2 $\beta$ A status (HR 2.1, 95\% CI 1.3-3.4, $p=0.001$ ), number of additional antibodies (HR $1.9,95 \%$ CI 1.1-3.1, $p=0.01$ ), and age (HR $0.965,95 \% \mathrm{CI}$ $0.933-0.997, p=0.02$ ), but not IA2A titre.

Of all ENDIT participants who developed diabetes, 79\% were IA2A-positive and 59\% were positive for both IA2A and IA $2 \beta A$.

\section{Discussion}

Diabetes risk in islet autoantibody-positive relatives is reflected by the breadth of the autoantibody response and b

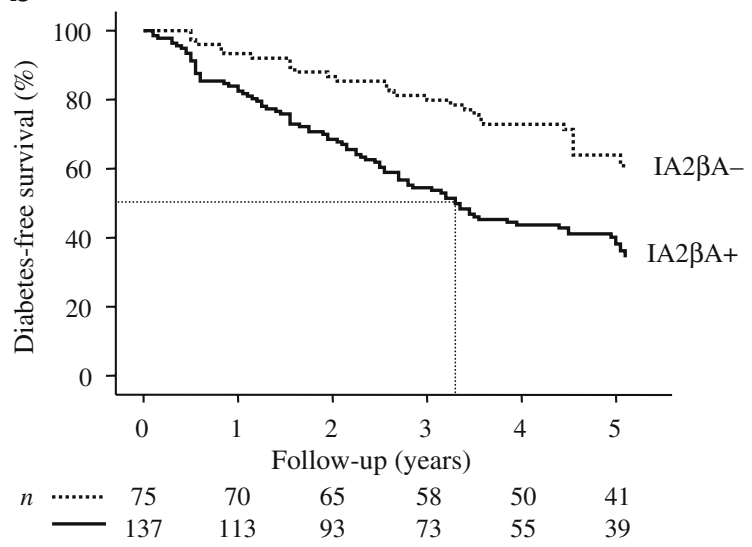

d

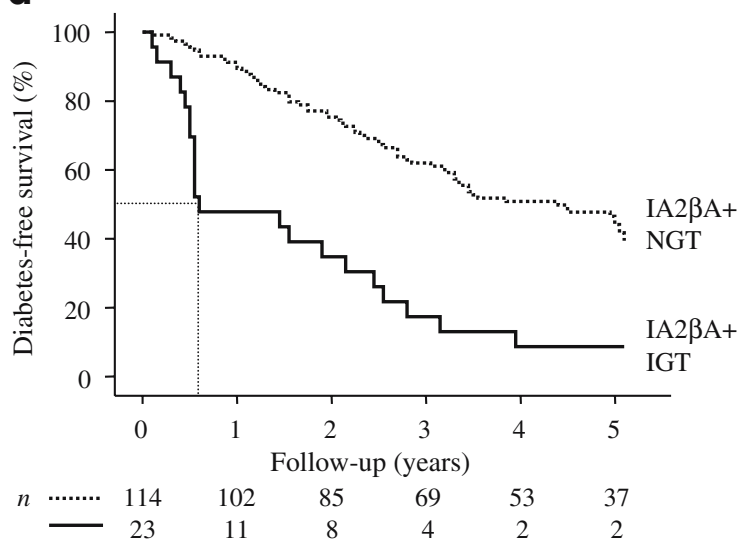

FPIR on IVGTT $(p<0.0001 ; \mathbf{c})$ or IGT on OGTT $(p<0.0001 ; \mathbf{d})$. Thin dotted lines indicate follow-up time at $50 \%$ progression to diabetes. NGT, normal glucose tolerance

the target antigens involved $[1,3,4,8]$. We have shown that autoantibodies to the IA- $2 \beta$ antigen detected at study entry were associated with very high diabetes risk, and that testing of IA2 $\beta \mathrm{A}$ in ICA/IA2A-positive relatives could further stratify their risk. IA $2 \beta A$ were particularly effective in identifying relatives who progressed to disease among those with ICA plus three additional biochemical autoantibodies (IA2A, IAA and GADA). Moreover, IA $2 \beta A$ compared favourably with the IVGTT in identifying individuals at high risk and achieved similar sensitivity [8], while combining IA2 $\beta$ A determination and IVGTT with standard antibody testing allowed identification of relatives with $>90 \% 5$ year risk.

The strengths of this study are the large number of wellcharacterised islet autoantibody-positive relatives in our cohort, the completeness of follow-up ( $88 \%$ of participants), and the broad inclusion criteria used in ENDIT. A limitation is that the participants were pre-selected on the basis of confirmed high-titre ICA, which are not likely to be used to select participants for future intervention trials, and absolute risks may not reflect those in populations with 
Table 1 Diabetes risk in ICA/ IA2A-positive relatives with respect to IA $2 \beta A$ status, number of additional islet autoantibodies, and metabolic markers a ICA/IA2A-positive, and IAA/ GADA-negative ${ }^{\mathrm{b}}$ ICA/IA2A-positive, and IAApositive or GADA-positive

${ }^{\mathrm{c}}$ ICA/IA2A-positive, and IAA/ GADA-positive

$\mathrm{Ab}$, antibody; NGT, normal glucose tolerance

\begin{tabular}{|c|c|c|c|c|}
\hline Additional characteristics & $\begin{array}{l}\text { Number in } \\
\text { group }\end{array}$ & $\begin{array}{l}\text { Number of } \\
\text { events }\end{array}$ & $\begin{array}{l}5 \text { year risk }(\%) \\
(95 \% \mathrm{CI})\end{array}$ & $p$ value \\
\hline \multicolumn{5}{|l|}{ IA $2 \beta A$ status } \\
\hline IA $2 \beta A$-negative & 75 & 28 & $39(28-50)$ & \multirow[t]{2}{*}{0.0002} \\
\hline IA $2 \beta A$-positive & 137 & 85 & $65(57-73)$ & \\
\hline \multicolumn{5}{|l|}{ Antibody number plus IA $2 \beta A$ status } \\
\hline \multicolumn{5}{|l|}{ 2Ab-positive ${ }^{\mathrm{a}}$} \\
\hline IA $2 \beta A$-negative & 8 & 0 & & \\
\hline IA $2 \beta A$-positive & 1 & 0 & & \\
\hline \multicolumn{5}{|l|}{ 3Ab-positive ${ }^{\mathrm{b}}$} \\
\hline IA $2 \beta A$-negative & 20 & 4 & $21(3-39)$ & \multirow[t]{2}{*}{0.15} \\
\hline IA $2 \beta$ A-positive & 35 & 14 & $44(26-62)$ & \\
\hline \multicolumn{5}{|l|}{$4 \mathrm{Ab}-$ positive $^{\mathrm{c}}$} \\
\hline IA $2 \beta A$-negative & 47 & 24 & $52(37-67)$ & \multirow[t]{2}{*}{0.003} \\
\hline IA $2 \beta A$-positive & 101 & 71 & $72(63-81)$ & \\
\hline \multicolumn{5}{|l|}{ IVGTT plus IA $2 \beta A$ status } \\
\hline \multicolumn{5}{|l|}{ Normal FPIR } \\
\hline IA $2 \beta A$-negative & 33 & 8 & $25(10-40)$ & \multirow[t]{2}{*}{0.07} \\
\hline IA $2 \beta$ A-positive & 66 & 27 & $44(31-57)$ & \\
\hline \multicolumn{5}{|l|}{ Low FPIR } \\
\hline IA $2 \beta$ A negative & 34 & 16 & $49(32-66)$ & \multirow[t]{2}{*}{$<0.0001$} \\
\hline IA $2 \beta$ A positive & 58 & 49 & $88(79-97)$ & \\
\hline \multicolumn{5}{|l|}{ OGTT plus IA2 $\beta$ A status } \\
\hline \multicolumn{5}{|l|}{ NGT } \\
\hline IA $2 \beta A$-negative & 69 & 23 & $35(23-47)$ & \multirow[t]{2}{*}{0.001} \\
\hline IA $2 \beta A$-positive & 114 & 64 & $59(49-69)$ & \\
\hline \multicolumn{5}{|l|}{ IGT } \\
\hline IA $2 \beta A$-negative & 6 & 5 & $83(53-100)$ & \multirow[t]{2}{*}{0.13} \\
\hline IA $2 \beta$ A-positive & 23 & 21 & $91(79-100)$ & \\
\hline \multicolumn{5}{|l|}{$\begin{array}{l}\text { Four autoantibodies plus IA } 2 \beta \text { A plus impaired } \\
\text { metabolic function }\end{array}$} \\
\hline 4Ab-positive ${ }^{c}$, IA2 $\beta A$-positive, with low FPIR & 45 & 41 & $94(86-100)$ & \\
\hline $4 \mathrm{Ab}$-positive ${ }^{\mathrm{c}}$, IA $2 \beta \mathrm{A}$-positive, with IGT & 21 & 20 & $95(86-100)$ & \\
\hline
\end{tabular}

lower overall risk. Participants were also selected for IA2A positivity, on the assumption that diabetes-relevant IA $2 \beta A$ are found only in the presence of IA2A. We are not therefore able to assess the impact of IA $2 \beta A$ in ICAnegative individuals or ICA-positive relatives who do not have IA2A. Further studies are required to determine whether our assumption that IA2 $\beta A$ testing is only necessary in IA2A-positive relatives is valid, and whether it is essential to measure the four other autoantibodies as well as IA $2 \beta A$. Furthermore, the small number of relatives in this study with only two antibody markers (ICA and IA2A) means that we did not have adequate power to evaluate the additional benefit of IA $2 \beta A$ testing in this subgroup.

Other studies have used antibody screening and IVGTT for risk assessment in relatives [10]. We have previously suggested alternative strategies based on islet autoantibody characteristics such as titre, epitope and IgG subclass binding, and antibody affinity that could stratify diabetes risk from $<10 \%$ up to $>90 \%$ in smaller cohorts of relatives $[1,5,6]$. However, some of these models are labour intensive and not in general use. In contrast, IA $2 \beta A$ are relatively easy to measure and have recently been evaluated in an international workshop by the Diabetes Antibody Standarisation Program (DASP). When added to IA2A, IA $2 \beta A$ identified $53 \%$ of all ENDIT participants who progressed to diabetes, with an associated 5 year risk of $65 \%$ on the basis of testing a single sample. In comparison, a low FPIR at baseline, a more demanding and costly test, conferred a $55 \% 5$ year risk and identified $57 \%$ of future cases [8]. Risks further increased to $72 \%$ in those IA $2 \beta A$-positive relatives with four additional antibodies (65\% of cases), and to $94 \%$ if FPIR was also low (at least $26 \%$ of cases).

In summary, several strategies can be used to stage pretype 1 diabetes in antibody-positive relatives. IA2 $\beta \mathrm{A}$ testing of a single sample offers a promising and flexible method of identifying those at high risk of relatively rapid progression to disease that also picks up a large proportion of future patients. Depending on the level of risk required for inclusion in a trial, a potential strategy might be to perform initial screening for IA2A (Fig. 1a), followed by measurement of IA2 $\beta \mathrm{A}$ in those who are IA2A-positive 
(Fig. 1b), with IVGTT (Fig. 1c) or OGTT (Fig. 1d) used to identify the subgroup of IA $2 \beta A$-positive relatives with the highest risk of rapid progression. Strategies based on IA2 $\beta A$ plus a small number of additional markers therefore provide the basis for relatively simple but highly effective identification of relatives at highest risk, and IA $2 \beta$ A should be included in the screening protocols for future type 1 diabetes intervention trials.

Acknowledgements ENDIT received support from the European Union (grants PL920957 and PL950771), the Juvenile Diabetes Research Foundation (4-2000-943), and Novo Nordisk. Participating centres are listed in the Electronic supplementary material. P. Achenbach was supported by Juvenile Diabetes Research Foundation (11-20051117). The authors would like to thank N. Grober and S. Krause for technical support.

Duality of interest The authors declare that there is no duality of interest associated with this manuscript.

\section{References}

1. Achenbach P, Warncke K, Reiter J et al (2004) Stratification of type 1 diabetes risk on the basis of islet autoantibody characteristics. Diabetes 53:384-392

2. Bingley PJ, Bonifacio E, Williams AJ, Genovese S, Bottazzo GF, Gale EAM (1997) Prediction of IDDM in the general population: strategies based on combinations of autoantibody markers. Diabetes 46:1701-1710

3. Decochez K, De Leeuw IH, Keymeulen B et al (2002) IA-2 autoantibodies predict impending type I diabetes in siblings of patients. Diabetologia 45:1658-1666

4. Krischer JP, Cuthbertson DD, Yu L et al (2003) Screening strategies for the identification of multiple antibody-positive relatives of individuals with type 1 diabetes. J Clin Endocrinol Metab 88:103-108

5. Achenbach P, Koczwara K, Knopff A, Naserke H, Ziegler AG, Bonifacio E (2004) Mature high-affinity immune responses to (pro)insulin anticipate the autoimmune cascade that leads to type 1 diabetes. J Clin Invest 114:589-597

6. Mayr A, Schlosser M, Grober N et al (2007) GAD Autoantibody affinity and epitope specificity identify distinct immunization profiles in children at risk for type 1 diabetes. Diabetes 56:15271533

7. Gale EAM, Bingley PJ, Emmett CL, Collier T (2004) European Nicotinamide Diabetes Intervention Trial (ENDIT): a randomised controlled trial of intervention before the onset of type 1 diabetes. Lancet 363:925-931

8. Bingley PJ, Gale EAM (2006) Progression to type 1 diabetes in islet cell antibody-positive relatives in the European Nicotinamide Diabetes Intervention Trial: the role of additional immune, genetic and metabolic markers of risk. Diabetologia 49:881-890

9. Bonifacio E, Lampasona V, Bingley PJ (1998) IA-2 (islet cell antigen 512) is the primary target of humoral autoimmunity against type 1 diabetes-associated tyrosine phosphatase autoantigens. J Immunol 161:2648-2654

10. Diabetes Prevention Trial-Type 1 Study Group (2002) Effects of insulin in relatives of patients with type 1 diabetes mellitus. $\mathrm{N}$ Engl J Med 346:1685-1691 